\title{
Programa de rehabilitación respiratoria infantil en un hospital público de Chile
} Pediatric pulmonary rehabilitation program at a Chilean public hospital

\author{
Klgo. Gerardo Torres ${ }^{a, b}$, Dr. Iván Rodríguez-Núñez ${ }^{b}$, Dr. Daniel Zenteno ${ }^{a, c}$, \\ Klga. Ximena Navarro ${ }^{a}$ Klga. Kimberly Medina ${ }^{b}$ y Dr. Jaime Tapia ${ }^{a}$
}

\section{RESUMEN}

Introducción. La rehabilitación respiratoria infantiles relevanteenel manejodeenfermedades respiratorias crónicas. En Chile, se desarrolla solo en algunos hospitales.

Objetivo. Describir los resultados de un programa de rehabilitación respiratoria infantil tras 3 meses de rehabilitación.

Metodología. Estudio retrospectivo de los pacientes con enfermedad pulmonar crónica, enfermedad neuromuscular y otras patologías crónicas admitidos desde junio de 2011 a junio de 2017. Se realizó evaluación física general, evaluación de musculatura respiratoria y espirometría. Se planificó entrenamiento físico general y entrenamiento muscular respiratorio, con protocolos institucional, mixtoy domiciliario. Resultados. Ingresaron al programa 156 pacientes $(11,45 \pm 3,55$ años $), 68$ con enfermedad pulmonar crónica $(11,56 \pm 4,6$ años, el $43,5 \%), 45$ con enfermedad neuromuscular (11,4 $\pm 3,7$ años, el $28,8 \%$ ) y 43 con patologías crónicas diversas (11,31 $\pm 3,9$ años). Del total, se entrenaron 102 (el 65,4\%); dirigido $(\mathrm{n}=27)$, mixto $(\mathrm{n}=23)$ y domiciliario $(\mathrm{n}=50)$ con adherencia del $69 \%$, del $87,5 \%$ y del $70 \%$, respectivamente. Aumentó el $19,3 \%$ la presión inspiratoria máxima $(p=0,001)$, el $14,5 \%$ la presión espiratoria máxima $(\mathrm{p}=0,001)$, el $12,9 \%$ la capacidad vital forzada $(p=0,001)$, el $11,6 \%$ el volumen espiratorio forzado al primer segundo $(p=0,004)$ y 17,6 metros en el test de marcha de 6 minutos $(p=0,036)$ tras 3 meses de rehabilitación.

Conclusiones. El protocolo de intervención incrementó el estatus funcional cardiorrespiratorio. La adherencia fue superior al $65 \%$.

Palabras clave: fuerza muscular, enfermedades neuromusculares, enfermedades pulmonares, rehabilitación, pediatría.

http: / / dx.doi.org/10.5546/ aap.2019.e576

Texto completo en inglés:

http:/ / dx.doi.org/10.5546/aap.2019.eng.e576

Correspondencia:

Lic. Gerardo Torres:

klgo.gerardo.torres@ gmail.com

Financiamiento:

Ninguno.

Conflicto de intereses:

Ninguno que declarar.

Recibido: 17-7-2018

Aceptado: 8-5-2019
Cómo citar: Torres G, Rodríguez-Núñez I, Zenteno D, Navarro X, et al. Programa de rehabilitación Arch Argent Pediatr 2019;117(6):e576-e583.

\section{GLOSARIO}

CVF: capacidad vital forzada.

DR: distancia recorrida.

EFG: entrenamiento físico general.

EMR: entrenamiento muscular respiratorio.

ENM: enfermedad neuromuscular. EPC: enfermedad pulmonar crónica. FC: frecuencia cardíaca.

FEF25-75: flujo espiratorio medio.

FEM: flujo espiratorio máximo.

FMT: flujo máximo de tos.

HGGB: Hospital Dr. Guillermo Grant

Benavente.

Pemax: presión espiratoria máxima.

Pimax: presión inspiratoria máxima.

RR: rehabilitación respiratoria.

TM6: test de marcha de 6 minutos.

VEF1: volumen espiratorio forzado

al primer segundo.

\section{INTRODUCCIÓN}

La rehabilitación respiratoria (RR) es una intervención interdisciplinaria, dirigida a pacientes con enfermedades crónicas. ${ }^{1} \mathrm{Su}$ objetivo es reducir síntomas, optimizar el estado funcional, aumentar la participación social y reducir los gastos en salud., ${ }^{2,3}$

El desarrollo de un programa de RR implica la articulación de estrategias de evaluación, el tratamiento y el seguimiento de pacientes, considerando la magnitud del deterioro funcional secundario al daño pulmonar y / o enfermedad neuromuscular (ENM), así como aspectos psicosociales de cada paciente. ${ }^{3,4}$

Actualmente, existen consensos y guías basadas en evidencia que han permitido protocolizar intervenciones asociadas con la evaluación y la rehabilitación de pacientes subsidiarios de RR y han establecido 
estándares mínimos para desarrollar este tipo de intervenciones en el contexto clínico. ${ }^{1-3}$ En Chile, son escasos los centros públicos que han desarrollado una base administrativa que permita la implementación de RR en los pacientes pediátricos con enfermedades crónicas. Entre los existentes, se encuentra el Programa de Rehabilitación Respiratoria Infantil del Hospital Dr. Guillermo Grant Benavente (HGGB) de Concepción. Este incorpora estrategias específicas de evaluación, rehabilitación y seguimiento, según las características biodemográficas y los aspectos fisiopatológicos de la enfermedad de cada paciente.

El rendimiento clínico del programa no ha sido reportado hasta la fecha, por lo que el objetivo de este estudio fue describir sus resultados tras 3 meses de rehabilitación.

\section{MATERIAL Y MÉTODO}

Diseño de estudio: Serie retrospectiva de casos.

Muestra de estudio: Todos los pacientes con enfermedad pulmonar crónica (EPC), ENM y otras patologías crónicas, admitidos desde junio de 2011 a junio de 2017 en el Programa de Rehabilitación Respiratoria Infantil del HGGB. Se excluyeron los pacientes con déficit cognitivo, menores de 5 años y con cuadro respiratorio agudo durante las 4 semanas previas al ingreso al programa.

\section{Protocolo de rehabilitación respiratoria infantil}

El protocolo de RR infantil del HGGB consta de intervenciones dirigidas a la evaluación y al tratamiento de pacientes con enfermedades crónicas (Figura 1).

FIGURA 1. Flujograma de derivación y atención del Policlinico de Rehabilitación Respiratoria Infantil, Hospital Dr. Guillermo Grant Benavente de Concepción

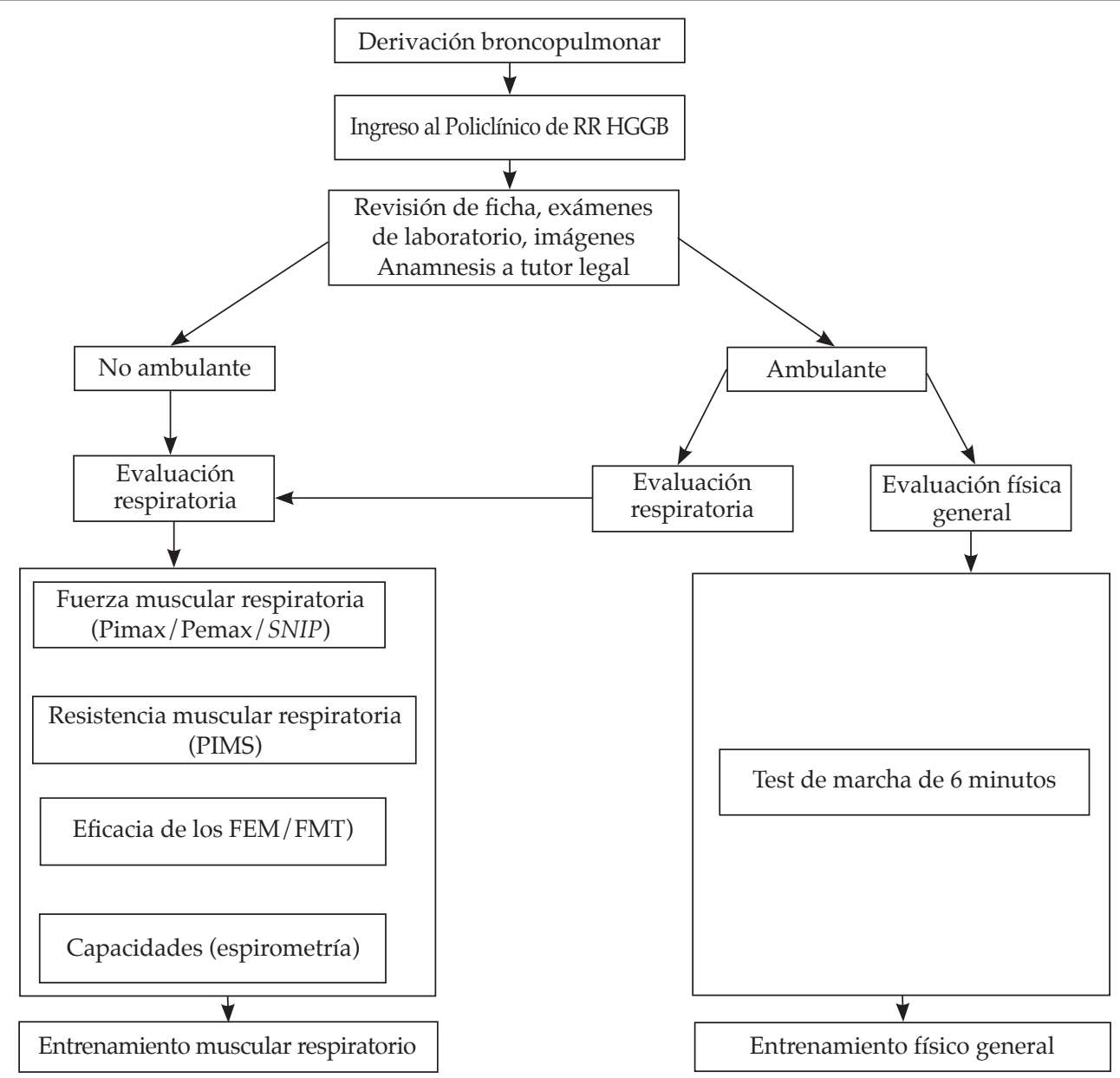

RR: rehabilitación respiratoria; HGGB: Hospital Dr. Guillermo Grant Benavente; Pimax: presión inspiratoria máxima; Pemax: presión espiratoria máxima; SNIP: presión nasal de succión; PIMS: presión inspiratoria máxima sostenida; FEM: flujo espiratorio máximo; FMT: flujo máximo tusígeno. 


\section{Protocolo de evaluación}

Se consideró edad, género, estatura y peso, medidos con cinta métrica y balanza análoga durante la admisión al programa. En los pacientes ambulantes, se realizó el test de marcha de 6 minutos (TM6), la evaluación de la presión inspiratoria máxima (Pimax), la presión espiratoria máxima (Pemax), flujometría y espirometría. En los pacientes no ambulantes, solo se realizó la evaluación de la Pimax, la Pemax, flujometría y espirometría. El padre o cuidador legal de cada niño firmó un consentimiento informado y los mayores de 12 años firmaron el asentimiento informado para el ingreso al programa y el uso de datos con fines científicos.

TM6: Aplicado según las normas de la American Thoracic Society. ${ }^{5}$ Se registró en una base de datos la distancia total recorrida (DR), frecuencia cardíaca (FC) de reposo y final del TM6 (oxímetro de pulso Choicemmed $^{\circledR}$ ), y percepción de esfuerzo con escala EPInfant, antes y después de la prueba. ${ }^{6} \mathrm{La}$ DR se registró en valores absolutos $(\mathrm{m})$ y porcentajes según los valores predictivos chilenos publicados por Gatica et al. ${ }^{7}$

Evaluación de la fuerza muscular respiratoria: La Pimax y la Pemax se midieron según protocolos de la European Respiratory Society y la American Thoracic Society. ${ }^{8}$ Se realizaron diez intentos, y se consideró el mejor valor de tres intentos aceptables y reproducibles con variabilidad $<5 \%$ y descanso de 30 segundos entre cada intento. Los resultados se expresaron en valores absolutos $\left(\mathrm{cmH}_{2} \mathrm{O}\right)$ y porcentajes, según los valores predictivos chilenos de Contreras et al. ${ }^{9}$ Las mediciones se realizaron usando un vacuómetro digital MicroRPM (Carefusion $^{\circledR}$ ).

Evaluación de la función pulmonar: Se evaluó mediante espirometría, según el protocolo establecido por la European Respiratory Society y la American Thoracic Society. ${ }^{10}$ Los parámetros registrados fueron el volumen espiratorio forzado al primer segundo (VEF1), la capacidad vital forzada (CVF), la relación VEF1 / CVF y el flujo espiratorio medio (FEF25-75). Los resultados fueron expresados en valores absolutos y porcentajes del valor predictivo según Knudson et ál., utilizándose un espirómetro portátil (Pony FX, Cosmed ${ }^{\circledR}$ ).

Evaluación de flujometría: El flujo espiratorio máximo (FEM) y el flujo máximo de tos (FMT) fueron medidos con un flujómetro (MiniWright $^{\circledR}$ ). El FEM se desarrolló con el sujeto de pie, utilizando una pinza nasal, que debía soplar con máxima fuerza desde la capacidad pulmonar total. Se realizó un máximo de ocho intentos; se registró el valor más alto reproducible en tres intentos con una diferencia no mayor del $10 \%$ entre cada uno de ellos. ${ }^{11}$ El FMT se midió con el sujeto sentado desde inspiración profunda y máximo esfuerzo de tos a través del flujómetro, y fue expresado en litros/minutos.

\section{Protocolo de intervención}

Los pacientes con valores inferiores al predicho en las pruebas realizadas ingresaron al protocolo de entrenamiento.

El entrenamiento físico general (EFG) se realizó al $60 \%$ de FC de reserva, con modalidad continua, 2-3 veces por semana, por 30 minutos. El desarrollado en el Hospital empleó un treadmill (Life Fitness ${ }^{\circledR}$ T9i) y, en el domicilio, se efectuó tras una consejería dirigida a educar al padre respecto a la dosificación de la intensidad y a la modalidad de ejercicio en el contexto domiciliario.

Además, el entrenamiento muscular respiratorio (EMR) se llevó a cabo con una válvula umbral (Threshold IMT, Philips Respironics ${ }^{\circledR}$ ). Así, el EMR inspiratorio se realizó con una carga equivalente al $30 \%$ de la Pimax, en 3 series de 3 minutos de trabajo y 1 minuto de descanso. El EMR espiratorio se realizó en 3 series de 15 a 20 repeticiones al $50 \%$ de la Pemax, con 1 minuto de descanso, que debió ser realizado 5 veces por semana tanto en el policlínico de rehabilitación como en el domicilio, según los diversos consensos. ${ }^{1,12,13}$

Seguimiento y adherencia: Para el seguimiento durante la RR, se establecieron 3 estrategias de acompañamiento de manera individualizada, según las variables geográficas y socioeconómicas: (a) Protocolo dirigido (evaluaciones, EMR y EFG 2-3 veces por semana en el policlínico de RR hospital-); (b) protocolo mixto (EMR domiciliario y 2-3 controles al mes, con EFG tanto en el hospital como en el domicilio); y (c) protocolo domiciliario no supervisado (EMR domiciliario, 1-2 controles cada 6 meses en el policlínico y EFG realizado íntegramente en el ambiente domiciliario). Los pacientes que poseían mejor acceso al centro hospitalario y factibilidad económica realizaron protocolos dirigidos o mixtos; mientras que quienes mostraron lo contrario fueron incluidos en protocolos domiciliarios.

Para determinar los resultados del protocolo, se consideraron los datos de las variables de interés tras 12 semanas del ingreso al programa de RR. 
La adherencia al EMR se estableció de acuerdo con la información proporcionada por el tutor/padre/madre del paciente. Cuando el entrenamiento fue realizado 5 días por semana, se consideró una adherencia adecuada. Por su parte, la adherencia al EFG se estableció solamente en el grupo que suscribió al protocolo dirigido, y se consideró adherencia adecuada cuando el paciente asistió ininterrumpidamente a sesiones programadas por, al menos, 8 semanas.

Análisis estadístico: Se realizó una estadística descriptiva con cálculo de promedio y desviación estándar para variables cuantitativas y porcentaje para variables cualitativas.

Se analizaron las características generales de los sujetos con EPC, ENM y otras enfermedades crónicas, y se desarrolló el análisis de la varianza (analysis of variance; ANOVA, por sus siglas en inglés) de un factor para las variables biodemográficas y el test de chi cuadrado para la variable género.
Se compararon los grupos de pacientes con EPC y ENM; se realizó el test $t$ de Student para muestras pareadas y Wilcoxon para comparar evaluaciones pre y pos protocolo de intervención; además, $U$ de Mann-Whitney y $t$ de Student para las muestras independientes al analizar entre ENM y EPC.

El resultado del entrenamiento se midió con el análisis de las diferencias entre los valores prey pos- de las variables evaluadas. El análisis se realizó con el paquete estadístico SPSS v.23.0 y se consideró significativo un valor de $\mathrm{p}<0,05$.

Consideraciones éticas: El estudio fue aprobado por el Comité Ético Científico del HGGB de Concepción.

\section{RESULTADOS}

En el período considerado, ingresaron 156 pacientes; 102 (el 65,4 \%) mostraron valores inferiores a los predichos en las pruebas evaluadas, por lo que realizaron algún tipo de entrenamiento.

TABla 1. Características generales de los grupos estudiados, expresadas en media \pm desviación estándar

\begin{tabular}{lccccc}
\hline Variables & Total & ENM & $\begin{array}{c}\text { EPC } \\
(\mathbf{n}=\mathbf{6 8})\end{array}$ & $\begin{array}{c}\text { Otras enfermedades crónicas } \\
(\mathbf{n}=\mathbf{4 3})\end{array}$ & Valor de $\boldsymbol{p}^{\mathbf{a}}$ \\
\hline Edad (años) & $11,5 \pm 3,6$ & $11,6 \pm 4,6$ & $11,4 \pm 3,7$ & $11,3 \pm 3,9$ & 0,371 \\
Género (M/F) & $91 / 65$ & $29 / 16$ & $41 / 27$ & $21 / 22$ & $0,302^{\mathrm{b}}$ \\
Peso (kg) & $40,1 \pm 17,6$ & $38,3 \pm 17,7$ & $39,8 \pm 15,3$ & $42,8 \pm 21,9$ & 0,577 \\
Estatura (cm) & $138,1 \pm 21,2$ & $134,2 \pm 19,0$ & $140,5 \pm 21,7$ & $138,2 \pm 22,8$ & 0,381 \\
IMC & $20,3 \pm 5,3$ & $20,6 \pm 5,9$ & $19,6 \pm 4,1$ & $21,5 \pm 6,5$ & 0,384 \\
\hline
\end{tabular}

IMC: índice de masa corporal; M: masculino; F: femenino; ENM: enfermedad neuromuscular; EPC: enfermedad pulmonar crónica. a ANOVA de un factor; ${ }^{\mathrm{b}}$ test de $\mathrm{chi}^{2}$.

TABLA 2. Evaluación inicial de la función muscular respiratoria, flujometría, espirometría y test de marcha de 6 minutos del grupo total y subgrupos con enfermedad neuromuscular y enfermedad pulmonar crónica, expresada en media \pm desviación estándar

\begin{tabular}{|c|c|c|c|c|}
\hline Variables & $\begin{array}{c}\text { Total } \\
(\mathrm{n}=156)\end{array}$ & $\begin{array}{c}\text { ENM } \\
(n=45)\end{array}$ & $\begin{array}{c}\text { EPC } \\
(n=68)\end{array}$ & Valor de $p^{\mathrm{b}}$ \\
\hline CVF (L) & $1,91 \pm 0,95$ & $1,68 \pm 0,69$ & $2,20 \pm 1,06$ & 0,083 \\
\hline $\operatorname{CVF}(\%)$ & $87,7 \pm 27,7$ & $84,9 \pm 33,5$ & $92,7 \pm 21,6$ & 0,382 \\
\hline VEF1 (L) & $1,51 \pm 0,70$ & $1,51 \pm 0,54$ & $1,63 \pm 0,79$ & $0,512^{\mathrm{a}}$ \\
\hline VEF1 $(\%)$ & $77,9 \pm 25,8$ & $83,4 \pm 30,8$ & $76,1 \pm 21,7$ & $0,052^{\mathrm{a}}$ \\
\hline VEF1/CVF & $77,4 \pm 22,2$ & $89,6 \pm 8,3$ & $75,0 \pm 13,0$ & $0,001^{\star_{a}}$ \\
\hline FEF25-75 $(1 / \mathrm{min})$ & $1,62 \pm 0,79$ & $1,97 \pm 0,70$ & $1,49 \pm 0,87$ & $0,004^{*_{a}}$ \\
\hline FEF25-75 $(\%)$ & $66,7 \pm 33,5$ & $93,6 \pm 29,2$ & $53,7 \pm 31,9$ & $0,001^{*_{a}}$ \\
\hline Pimax $\left(\mathrm{cmH}_{2} \mathrm{O}\right)$ & $59,6 \pm 20,4$ & $55,3 \pm 19,7$ & $66,2 \pm 19,0$ & $0,010^{*}$ \\
\hline $\operatorname{Pimax}(\%)$ & $57,4 \pm 20,5$ & $51,7 \pm 17,5$ & $64,3 \pm 19,8$ & $0,006^{*}$ \\
\hline Pemax $\left(\mathrm{cmH}_{2} \mathrm{O}\right)$ & $58,0 \pm 24,5$ & $42,6 \pm 18,4$ & $70,5 \pm 21,6$ & $0,001^{*}$ \\
\hline Pemax $(\%)$ & $43,7 \pm 18,0$ & $32,2 \pm 14,4$ & $52,6 \pm 17,0$ & $0,001^{*_{a}}$ \\
\hline $\operatorname{PEF}(1 / \min )$ & $203,2 \pm 102,5$ & $150,36 \pm 51,3$ & $230,3 \pm 107,2$ & 0,331 \\
\hline PEFt (1/min) & $198 \pm 128,2$ & $165,0 \pm 56,4$ & $217,7 \pm 84,2$ & 0,318 \\
\hline TM6 (m) & $513,9 \pm 132,2$ & $359,8 \pm 138,2$ & $564,6 \pm 89,7$ & $0,001^{*}$ \\
\hline TM6 (\%) & $81,5 \pm 16,7$ & $62,3 \pm 18,9$ & $67,0 \pm 20,7$ & $0,001^{*}$ \\
\hline
\end{tabular}

Pimax: presión inspiratoria máxima; Pemax: presión espiratoria máxima; PEF: flujo espiratorio máximo; PEFt: flujo tusígeno máximo; TM6: test de marcha de 6 minutos; ENM: enfermedad neuromuscular; EPC: enfermedad pulmonar crónica.

${ }^{*}$ Diferencia estadísticamente significativa con $p<0,05$; ${ }^{a}$ test U de Mann-Whitney; ${ }^{b}$ test $t$ de Student. 
No se observaron diferencias entre los grupos de pacientes con EPC, ENM y otras enfermedades crónicas respecto a edad, género y variables antropométricas (Tabla 1). El grupo de ENM presentó, principalmente, distrofia muscular de Duchenne $(n=13)$, mielomeningocele $(n=10)$ $\mathrm{y}$ atrofia muscular espinal (AME) II $(\mathrm{n}=5)$; y el grupo de EPC presentó bronquiolitis obliterante $(n=27)$, fibrosis quística $(n=19)$ y asma $(n=15)$, entre otras. Las otras enfermedades crónicas fueron síndrome de apneas del sueño, obesidad, escoliosis grave y cardiopatías. La totalidad de la muestra no presentó insuficiencia respiratoria con requerimiento de oxígeno.

De los 102 pacientes entrenados, 38 realizaron solo EMR; 27, solo EFG; y 37, EMR/EFG. De los que hicieron EMR, 34 (el $45 \%$ ) fueron ENM, y 23 (el $31 \%), \operatorname{EPC}(\mathrm{p}=0,001)$, mientras que, de los que realizaron EFG, 12 (el $19 \%$ ) presentaron ENM, y $37(\mathrm{el} 58 \%)$, EPC ( $\mathrm{p}=0,001)$.

Respecto a la modalidad del entrenamiento, 27 pacientes (el 19,4\%) desarrollaron entrenamiento dirigido; 23 (el 16,5\%), entrenamiento mixto, y 50 (el 40,0\%), entrenamiento domiciliario, con un porcentaje de adherencia del $69,0 \%$, del $87,5 \%$ y del $70,0 \%$, respectivamente.

En la medición de la fuerza muscular respiratoria, ambos grupos presentaron la Pimax y la Pemax inferiores al valor normal teórico (Tabla 2). Se observó la existencia de un patrón ventilatorio obstructivo en los niños con EPC. Por su parte, los pacientes con ENM mostraron un patrón ventilatorio normal.

\section{Resultados del protocolo de intervención sobre los parámetros de función respiratoria y capacidad física general}

En la Figura 2, se observan los cambios tras el período de RR en la fuerza muscular respiratoria, flujometría y el test de marcha del grupo total entrenado $(n=102)$.

En la fuerza muscular respiratoria, se observó un aumento de $11,5 \mathrm{cmH}_{2} \mathrm{O}$ en la Pimax (el 19,3\%; $\mathrm{p}=0,001)$ y $8,41 \mathrm{cmH}_{2} \mathrm{O}$ en la Pemax (el 14,5\%; $\mathrm{p}=0,001)$. En la espirometría, la CVF aumentó en 0,246 litros (el 12,9\%; $\mathrm{p}=0,001$ ), y el VEF1, en 0,177 litros (el 11,6\%; p = 0,004) (Tabla 3). Por su parte, la distancia del TM6 aumentó en 17,6 metros (el 3,4 \%; $\mathrm{p}=0,036$ ).

Al realizar el análisis por grupo de ENM y EPC, los pacientes con ENM mejoraron en $7,66 \mathrm{cmH}_{2} \mathrm{O}$ la Pemax (el 17,9\%; $\left.\mathrm{p}=0,014\right)$ y en $22,21 /$ min el FMT (el 18,8 \%; $p=0,021)$. En el caso de los pacientes con EPC, mejoraron 0,396 litros
FIGURA 2. Gráficos de evaluación inicial y tras 3 meses del ingreso de la fuerza muscular respiratoria (A), flujometría (B) $y$ distancia del test de marcha de 6 minutos (C) del grupo total entrenado $(n=102)$

A

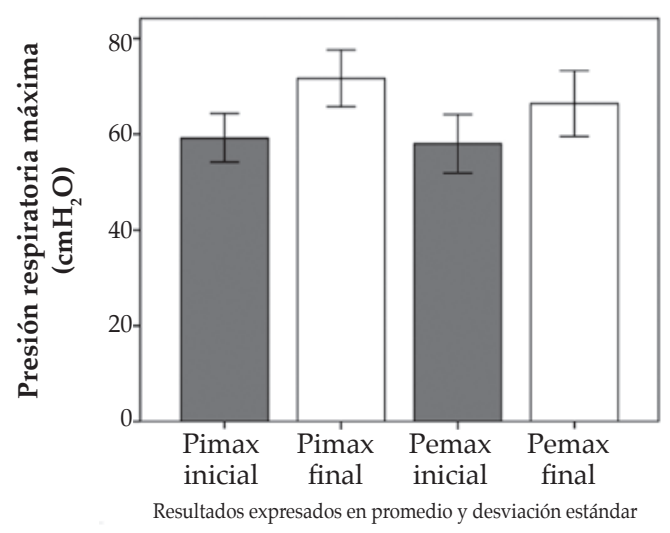

B

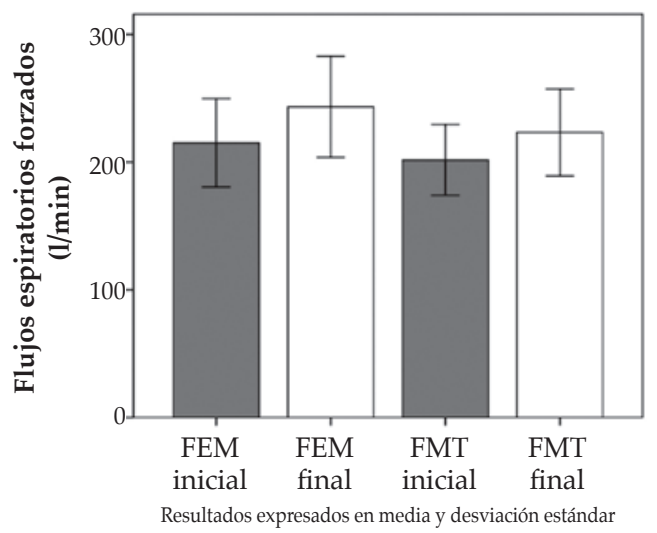

C

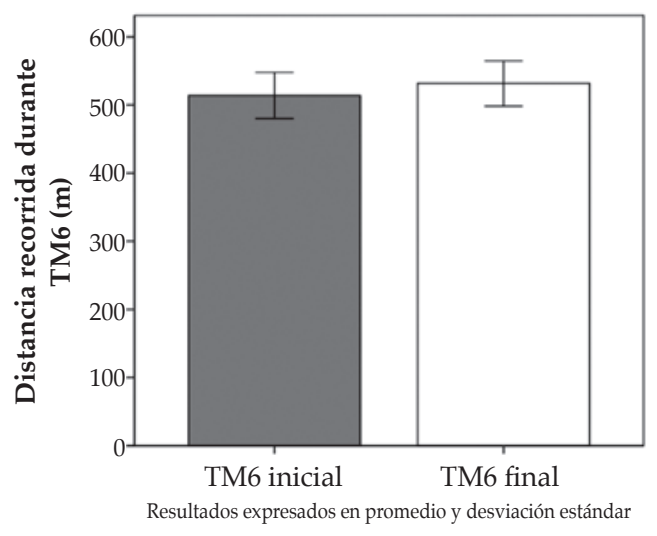

PIMAX: presión inspiratoria máxima; PEMAX: presión espiratoria máxima; FEM: flujo espiratorio máximo; FMT: flujo máximo tusígeno; TM6: test de marcha de 6 minutos.

del CVF (el 17,9\%; p = 0,001), 0,325 litros del VEF1 (el 19,9 \%; p =0,001) y 11,7 $\mathrm{cmH}_{2} \mathrm{O}$ de la Pimax (el 17,6\%; p =0,006) (Tabla 4). 


\section{DISCUSIÓN}

El objetivo del trabajo fue describir las características de un programa de RR dirigido a niños y adolescentes con enfermedades crónicas en un hospital público de Chile. Se observó que los pacientes experimentaron mejoras significativas en la fuerza muscular respiratoria, volúmenes y capacidades pulmonares, así como una mayor DR durante el TM6. Estos resultados concordaron con aquellos que habían revelado los beneficios del EMR y del EFG sobre la función de los músculos respiratorios, las variables espirométricas y el TM6. ${ }^{12-17}$

La totalidad de la muestra presentó edades similares, pues los criterios de inclusión al programa consideraban desde los 5 años con síntomas y limitaciones persistentes; ${ }^{1}$ mientras que la gravedad de la patología o las variables específicas de la función pulmonar no constituyeron criterios de inclusión al programa de RR, lo que permitió mantener o mejorar las capacidades cardiorrespiratorias antes de un deterioro más avanzado. El $65 \%$ de los pacientes adhirieron al programa, excepto 3 con ENM y 19 con EPC, que presentaban insuficiente adherencia a otros tratamientos médicos, mayor distancia del centro asistencial, regular control de su patología de base no existente antes del inicio de RR y situación socioeconómica que limitó su acceso, por lo que se debió modificar su estrategia de rehabilitación para mantener su adherencia.

En nuestro estudio, se observaron incrementos del $19,3 \%$ en la Pimax y del $14,5 \%$ en la Pemax, lo que concordó con Rodríguez et al., quienes observaron mejoras en la fuerza muscular respiratoria de los niños con ENM y EPC, luego de un protocolo domiciliario de EMR. ${ }^{18}$

TABLA 3. Evaluación espirométrica inicial y posterior a 3 meses del ingreso del grupo total entrenado $(n=102)$, expresada en media \pm desviación estándar

\begin{tabular}{lccc}
\hline Variables & Evaluación inicial & Evaluación a 3 meses & Valor de $p^{\text {b }}$ \\
\hline CVF $(1)$ & $1,91 \pm 0,95$ & $2,15 \pm 1,23$ & $0,001^{* a}$ \\
CVF (\%) & $87,7 \pm 27,7$ & $87,2 \pm 26,2$ & 0,847 \\
VEF1 $(1)$ & $1,51 \pm 0,70$ & $1,69 \pm 0,94$ & $0,004^{* a}$ \\
VEF1 (\%) & $77,9 \pm 25,8$ & $77,1 \pm 25,2$ & $0,664^{\mathrm{a}}$ \\
VEF1/CVF & $77,4 \pm 22,2$ & $75,7 \pm 18,5$ & $0,018^{* a}$ \\
FEF25-75 (1/min) & $1,62 \pm 0,79$ & $1,72 \pm 1,07$ & $0,328^{\mathrm{a}}$ \\
FEF25-75 (\%) & $66,7 \pm 33,5$ & $68,4 \pm 39,1$ & $0,636^{\mathrm{a}}$ \\
\hline
\end{tabular}

${ }^{*}$ Diferencias significativas con $p<0,05 ;{ }^{\text {a }}$ test de Wilcoxon.

TABLA 4. Evaluación inicial y posterior a 3 meses del ingreso de los grupos con enfermedad neuromuscular y enfermedad pulmonar crónica, expresada en media \pm desviación estándar

\begin{tabular}{|c|c|c|c|c|c|c|}
\hline \multirow[t]{2}{*}{ Variables } & \multicolumn{3}{|c|}{$\begin{array}{c}\text { ENM } \\
(n=35)\end{array}$} & \multicolumn{3}{|c|}{$\begin{array}{c}\text { EPC } \\
(n=49)\end{array}$} \\
\hline & Pre- & Pos- & Valor de $p$ & Pre- & Pos- & Valor de $p$ \\
\hline CVF (l) & $1,68 \pm 0,69$ & $1,71 \pm 0,74$ & 0,704 & $2,20 \pm 1,06$ & $2,60 \pm 1,40$ & $0,001^{*}$ \\
\hline CVF $(\%)$ & $84,9 \pm 33,5$ & $79,85 \pm 28,2$ & 0,252 & $92,7 \pm 21,6$ & $93,6 \pm 23,6$ & 0,748 \\
\hline VEF1 (1) & $1,51 \pm 0,54$ & $1,53 \pm 0,59$ & $0,530^{a}$ & $1,63 \pm 0,79$ & $1,96 \pm 1,08$ & $0,001^{* a}$ \\
\hline VEF1 (\%) & $83,4 \pm 30,8$ & $78,5 \pm 24,0$ & 0,267 & $76,1 \pm 21,7$ & $78,6 \pm 25,8$ & $0,676^{a}$ \\
\hline VEF1/CVF & $89,6 \pm 8,3$ & $88,7 \pm 8,5$ & 0,496 & $75,0 \pm 13,0$ & $71,7 \pm 13,0$ & $0,005^{*}$ \\
\hline FEF25-75 (1/ min $)$ & $1,97 \pm 0,70$ & $2,03 \pm 0,84$ & 0,606 & $1,49 \pm 0,87$ & $1,59 \pm 1,18$ & $0,566^{\mathrm{a}}$ \\
\hline FEF25-75 $(\%)$ & $93,6 \pm 29,2$ & $95,0 \pm 38,7$ & $0,433^{a}$ & $53,7 \pm 31,9$ & $56,8 \pm 35,6$ & $0,663^{\mathrm{a}}$ \\
\hline $\operatorname{Pimax}\left(\mathrm{cmH}_{2} \mathrm{O}\right)$ & $55,3 \pm 19,7$ & $61,6 \pm 20,8$ & 0,093 & $66,2 \pm 19,0$ & $77,8 \pm 21,8$ & $0,006^{*_{a}}$ \\
\hline $\operatorname{Pimax}(\%)$ & $51,7 \pm 17,5$ & $58,4 \pm 18,3$ & 0,093 & $64,3 \pm 19,8$ & $76,5 \pm 24,6$ & $0,009^{*}$ \\
\hline Pemax $\left(\mathrm{cmH}_{2} \mathrm{O}\right)$ & $42,6 \pm 18,4$ & $50,29 \pm 19,8$ & $0,014^{*}$ & $70,5 \pm 21,6$ & $75,7 \pm 21,6$ & 0,239 \\
\hline $\operatorname{Pemax}(\%)$ & $32,2 \pm 14,4$ & $37,0 \pm 15,4$ & $0,126^{a}$ & $52,6 \pm 17,0$ & $55,8 \pm 16,5$ & 0,291 \\
\hline $\operatorname{PEF}(1 / \mathrm{min})$ & $150,4 \pm 51,3$ & $178,5 \pm 53,4$ & $0,021^{*}$ & $230,3 \pm 107,2$ & $251,7 \pm 130,9$ & 0,062 \\
\hline PEFt (1/min) & $165,0 \pm 56,4$ & $165,0 \pm 62,5$ & 1,000 & $217,7 \pm 84,2$ & $248,5 \pm 108,5$ & 0,070 \\
\hline TM6 (m) & $359,8 \pm 138,2$ & $376,4 \pm 147,4$ & 0,445 & $564,6 \pm 89,7$ & $582,0 \pm 87,5$ & 0,073 \\
\hline TM6 (\%) & $62,3 \pm 18,9$ & $61,1 \pm 22,8$ & 0,742 & $67,0 \pm 20,7$ & $70,948 \pm 20,6$ & 0,150 \\
\hline
\end{tabular}

Pimax: presión inspiratoria máxima; Pemax: presión espiratoria máxima; PEF: flujo espiratorio máximo; PEFt: flujo tusígeno máximo; TM6: test de marcha de 6 minutos; ENM: enfermedad neuromuscular; EPC: enfermedad pulmonar crónica.

${ }^{*}$ Diferencias significativas con $p<0,05$; ${ }^{\text {a }}$ test de Wilcoxon. 
En el grupo total, existió un incremento en los parámetros de la función pulmonar posterior al protocolo del 12,9\% del valor absoluto de CVF y del 11,9\% del valor absoluto de VEF1. Estudios previos han mostrado resultados discrepantes respecto al impacto de los protocolos de RR sobre estas variables. Colom et al., mostraron que aumentaban los volúmenes espiratorios forzados, pero la CVF se incrementaba desproporcionadamente respecto al VEF1, y disminuía el índice VEF1/CVF. Por esto, no es posible descartar que los cambios observados puedan tener relación con fenómenos asociados al crecimiento de los niños o al aprendizaje de las pruebas de evaluación. ${ }^{19}$

Se observó un incremento del $3 \%$ en la DR durante el TM6 en la totalidad de la muestra, sin significancia estadística por subgrupos de patologías. Estos resultados concordaron con Rodríguez et al., ${ }^{20}$ en los pacientes con ENM, no así con los resultados de Latorre-Román et al., ${ }^{21}$ quienes observaron mejoras en la DR del TM6 de niños con asma.

De forma específica, en los pacientes con ENM, se han observado, tras 10 meses de entrenamiento, incrementos en la fuerza de los músculos inspiratorios y espiratorios equivalentes al $45,5 \%$ y al $37,5 \%$, respectivamente, ${ }^{18}$ que alcanzó una fase de meseta a los 10 meses de entrenamiento. ${ }^{12}$ Estos antecedentes discrepan de nuestros resultados, pues solo se observaron incrementos en la fuerza de los músculos espiratorios. Es probable que la reevaluación de la fuerza muscular respiratoria tras un breve período de EMR de 3 meses haya subestimado los resultados del protocolo ejecutado en nuestro Centro. Esta reevaluación precoz se debió a que muchos pacientes, a partir de los 3 meses, cambiaron estrategias de rehabilitación a protocolos mixtos o ambulatorios, lo que significó resultados más heterogéneos.

En nuestro estudio, los pacientes con ENM no mostraron cambios significativos en los parámetros espirométricos ni en la DR durante el TM6, tal como fue expresado en otros artículos. ${ }^{22-23}$ Esto concordó con los resultados del estudio de Rodríguez et al., ${ }^{20}$ realizado en pacientes con ENM progresiva, que no observaron incrementos en la DR durante el TM6; sin embargo, constataron una reducción significativa en la percepción de esfuerzo y fatiga de piernas, posterior a un protocolo de EFG. Se ha sugerido que las variables como calidad de vida y consumo de oxígeno experimentan mejoras significativas luego de intervenciones basadas en entrenamiento físico, sin evidencia de lesión muscular inducida por ejercicio. ${ }^{24}$

En pacientes con EPC, estudios confirman el impacto positivo del EMR, que muestran mejoras de la fuerza muscular respiratoria tras un protocolo de entrenamiento. ${ }^{13,15,25}$ Rodríguez et al., observaron variaciones del 33,3\% en la Pimax y del 33,3\% en la Pemax en los pacientes con EPC tras 10 meses de EMR. ${ }^{20}$ En nuestro estudio, los pacientes con EPC mostraron mejoras del 17,9 \% del valor absoluto de la Pimax.

Estos pacientes mostraron cambios favorables en las variables espirométricas. Se ha demostrado que el EFG previene la caída de la función pulmonar cuando los protocolos de rehabilitación se desarrollan a largo plazo. ${ }^{26-28}$

Nuestro estudio presenta limitaciones, tales como que la heterogeneidad de la población no permitió establecer conclusiones sobre el comportamiento de una patología específica, lo que generó la dispersión de los resultados. La ausencia de un grupo control y el enmascaramiento en las mediciones efectuadas impidieron descartar la potencial influencia del aprendizaje de los niños para realizar las pruebas, lo que pudo haber sobreestimado los resultados. Algunas de las asociaciones verificadas no reportaron significancia estadística; sin embargo, la magnitud absoluta de estas diferencias concordó con los resultados de estudios previamente realizados. En consecuencia, no se puede descartar que el tamaño muestral de esta serie de casos haya sido insuficiente para aminorar el riesgo de error de tipo 2 en algunos de los análisis realizados.

Finalmente, es posible concluir que los niños y adolescentes con enfermedades crónicas presentaron una menor capacidad funcional cardiorrespiratoria y aquellos que ingresaron al protocolo de rehabilitación lograron incrementar la fuerza de los músculos respiratorios y la capacidad física. Futuros estudios controlados deben ser desarrollados para confirmar estos resultados.

\section{REFERENCIAS}

1. Torres-Castro R, Zenteno D, Rodríguez-Núñez I, Villarroel $\mathrm{G}$, et al. Guías de rehabilitación respiratoria en niños con enfermedades respiratorias crónicas: Actualización 2016. Neumol Pediatr. 2016; 11(3):114-31.

2. Zenteno D, Puppo H, Vera R, Torres R, et al. Guías de rehabilitación para niños con enfermedades respiratorias crónicas. Neumol Pediatr. 2008; 3(supl):25-33.

3. Spruit MA, Singh SJ, Garvey C, ZuWallack R, et al. An official American Thoracic Society/European Respiratory 
Society statement: key concepts and advances in pulmonary rehabilitation. Am J Respir Crit Care Med. 2013; 188(8):e13-64.

4. Linares M, Sánchez I, Corrales R, Díaz A, et al. Pruebas de función pulmonar en el niño. Rev Chil Pediatr. 2000; 71(3):228-42.

5. ATS Committee on Proficiency Standards for Clinical Pulmonary Function Laboratories. ATS statement: guidelines for the six-minute walk test. Am J Respir Crit Care Med. 2002; 166(1):111-7.

6. Rodríguez I, Zenteno D, Cisternas L, Rodríguez P, et al. Construcción y evaluación de EPInfant: una escala para la medición del esfuerzo percibido en la población pediátrica. Arch Argent Pediatr. 2015; 113(6):550-7.

7. Gatica D, Puppo H, Villarroel G, San Martín I, et al. Valores de referencia del test de marcha de seis minutos en niños sanos. Rev Med Chile. 2012; 140(8):1014-21.

8. American Thoracic Society/European Respiratory Society. ATS/ERS Statement on respiratory muscle testing. Am J Respir Crit Care Med. 2002; 166(4):518-624.

9. Contreras I, Vidal F, Caussade S, Sánchez I, et al. Valores normales de presión inspiratoria y presión espiratoria máxima en niños y adolescentes chilenos sanos. Neumol Pediatr. 2007; 2(3):163.

10. Miller MR, Hankinson J, Brusasco V, Burgos F, et al. Standardisation of spirometry. Eur RespirJ. 2005;26(2):319-38.

11. Céspedes GJ, Gutiérrez CM, Oyarzún GM. Flujometría en la práctica de atención primaria. Rev Chil Enferm Respir. 2010; 26(1):47-8.

12. Koessler W, Wanke T, Winkler G, Nader A, et al. 2 Years' experience with inspiratory muscle training in patients with neuromuscular disorders. Chest. 2001; 120(3):765-9.

13. Houston BW, Mills N, Solis-Moya A. Inspiratory muscle training for cystic fibrosis. Cochrane Database Syst Rev. 2013; (11):CD006112.

14. De Jong W, Van Aalderen W, Kraan J, Koeter G, et al. Inspiratory muscle training in patients with cystic fibrosis. Respir Med. 2001; 95(1):31-6.

15. SilvaJ,PuppoH, VargasD, González R, et al. Entrenamiento muscular inspiratorio en niños secuelados por adenovirus. Rev Chil Enferm Respir. 1999; 15:239.

16. Gozal D, Thiriet P. Respiratory muscle training in neuromuscular disease: long-term effects on strength and load perception. Med Sci Sports Exerc. 1999; 31(11):1522-7.

17. Topin N, MateckiS, Le Bris S, RivierF, etal. Dose-dependent effect of individualized respiratory muscle training in children with Duchenne muscular dystrophy. Neuromuscul Disord. 2002; 12(6):576-83.

18. Rodríguez Núñez I, Zenteno Araos D, Manterola Delgado C. Effects of home-based respiratory muscle training in children and adolescent with chronic lung disease. J Bras Pneumol. 2014; 40(6):626-33.

19. Colom AJ, Maffey A, Garcia Bournissen F, Teper A. Pulmonary function of a paediatric cohort of patients with postinfectious bronchiolitis obliterans. A long term followup. Thorax. 2015; 70(2):169-74.

20. Rodríguez Núñez I, Fuentes SC, Rivas BC, Molina RF, et al. Rehabilitación respiratoria en el paciente neuromuscular: efectos sobre la tolerancia al ejercicio y musculatura respiratoria. Resultado de una serie de casos. Rev Chil Enferm Respir. 2013; 29(4):196-203.

21. Latorre-Román P, Navarro-Martínez V, García-Pinillos F. The effectiveness of an indoor intermittent training program for improving lung function, physical capacity, body composition and quality of life in children with asthma. $J$ Asthma. 2014; 51(5):544-51.

22. Khirani S, Colella M, Caldarelli V, Aubertin G, et al. Longitudinal course of lung function and respiratory muscle strength in spinal muscular atrophy type 2 and 3. Eur J Paediatr Neurol. 2013; 17(6):552-60.

23. KhiraniS, Ramirez A, Aubertin G, BouléM, etal. Respiratory muscle decline in Duchenne muscular dystrophy. Pediatr Pulmonol. 2014; 49(5):473-81.

24. Aboussouan LS. Mechanisms of exercise limitation and pulmonary rehabilitation for patients with neuromuscular disease. Chron Respir Dis. 2009; 6(4):231-49.

25. Taivassalo T, De Stefano N, Chen J, Karpati G, et al. Short $\square$ term aerobic training response in chronic myopathies. Muscle Nerve. 1999; 22(9):1239-43.

26. Lötters F, Van Tol B, Kwakkel G, Gosselink R. Effects of controlled inspiratory muscle training in patients with COPD: a meta-analysis. Eur Respir J. 2002; 20(3):570-6.

27. Van Doorn N. Exercise programs for children with cystic fibrosis: a systematic review of randomized controlled trials. Disabil Rehabil. 2010; 32(1):41-9.

28. Eichenberger PA, Diener SN, Kofmehl R, Spengler CM. Effects of exercise training on airway hyperreactivity in asthma: a systematic review and meta-analysis. Sports Med. 2013; 43(11):1157-70. 